\title{
Beam delivery system tuning and luminosity monitoring in the Compact Linear Collider
}

\author{
B. Dalena, J. Barranco, A. Latina, E. Marin, J. Pfingstner, D. Schulte, J. Snuverink, \\ R. Tomás, and G. Zamudio \\ CERN, Geneva, Switzerland \\ (Received 17 November 2011; published 30 May 2012)
}

\begin{abstract}
Emittance preservation in the beam delivery system (BDS) is one of the major challenges in the Compact Linear Collider (CLIC). The fast detuning of the final focus optics requires an on-line tuning procedure in order to keep luminosity close to the maximum. In this paper we discuss different tuning techniques to mitigate the displacement of magnets in the CLIC BDS and in particular in the final focus system. Some of them require a fast luminosity measurement. Here we study the possibility to use beambeam background processes at CLIC $3 \mathrm{TeV}$ c.m. energy as a fast luminosity signal. In particular, the hadron multiplicity in the detector region is investigated.
\end{abstract}

DOI: 10.1103/PhysRevSTAB.15.051006

PACS numbers: 41.75.Ht, 29.27.Fh, 41.60. $-\mathrm{m}$

\section{INTRODUCTION}

In order to achieve high luminosity in the Compact Linear Collider (CLIC) nanometer beam sizes at the interaction point (IP) are required. One of the major challenges of this future linear collider is the preservation of the transverse emittances from the damping ring to the IP. Amongst the most critical sources of emittance growth are static and dynamic imperfections along the main linac (ML) and the beam delivery system (BDS). These are the systems where the tolerances on magnet misalignment and errors are tighter $(\sim 10 \mu \mathrm{m}$ for the static and fraction of $\mathrm{nm}$ for the dynamic imperfections). The tuning of the static imperfections do not require any fast procedure except for the last part of the Final Focus System (FFS), when the push-pull scenario is considered. The tuning of the dynamic imperfections needs to be done at faster time scales, in order to keep luminosity stable during operation. Integrated simulations of ML and BDS including ground motion lead to a luminosity loss of the order of $10 \%$ after about 1 hour [1].

Traditionally, by the tuning of a linear collider, we understand the full correction procedure in order to bring the system to its ideal performance, as described in [2]. In this paper we discuss the mitigation against static magnet displacements in the BDS. Thus, in the following the word tuning will assume this meaning. The main reason to consider only magnet displacements is because of their relevance in the dynamic case, and common solutions can be adopted in the two cases. Moreover, we consider the BDS and in particular the FFS, due to the known difficulty to tune the system [3]. The origin of this behavior comes from the strong contribution of nonlinear terms in

Published by the American Physical Society under the terms of the Creative Commons Attribution 3.0 License. Further distribution of this work must maintain attribution to the author(s) and the published article's title, journal citation, and DOI. the design of the FFS optics [4] and the increasing difficulty in tuning the system with a very low $\beta$ function at the IP, as expected from simulations and empirically tested [5]. Furthermore, the integrated simulation of ML and BDS including ground motion shows that the source of the luminosity loss is the fast detuning of the FFS optics. In fact, the luminosity loss can be fully recovered by adding to the orbit control feedback the scan of precomputed orthogonal knobs [1]. The knobs and the first results achieved in the tuning of the BDS will be presented in Sec. II. The tuning procedures we studied exploit luminosity as a figure of merit. Therefore it is mandatory to have a method to estimate the luminosity. Note that the luminosity measurements needed by the tuning procedure are not necessarily a measurement of the luminosity value. For the purpose of this tuning it can be a signal whose rate scales like luminosity (with imperfections considered).

The measurement of luminosity in $e^{+} e^{-}$colliders is usually done by detecting radiative Bhabhas $\left(e^{+} e^{-} \rightarrow\right.$ $e^{+} e^{-} \gamma$ ) [6] in the detector's forward region. In CLIC at $3 \mathrm{TeV}$ c.m. energy there is no energy window where the electrons or positrons can be separated from the spent beam low energy tails or the incoherent pairs energy distribution, as shown in Fig. 1. The low angle Bhabhas have a lower event rate than radiative Bhabhas at the CLIC c.m. energy. Moreover, these methods need from 7 to 70 minutes in order to reach $1 \%$ precision in the measurement of the luminosity [7]. The fast detuning of the machine is then not compatible with this technique.

The possibility to use secondary particles emitted during the beam-beam interaction to monitor luminosity at CLIC has already been proposed [8]. In particular, the possibility to use the beamstrahlung photons as a fast luminosity signal has been exploited in [9]. The measurement of the beam sizes at the IP, using incoherent pairs both alone or in combination with beamstrahlung, has been explored in [10]. In the following, the results in terms of CLIC BDS performances are presented, according to the different 

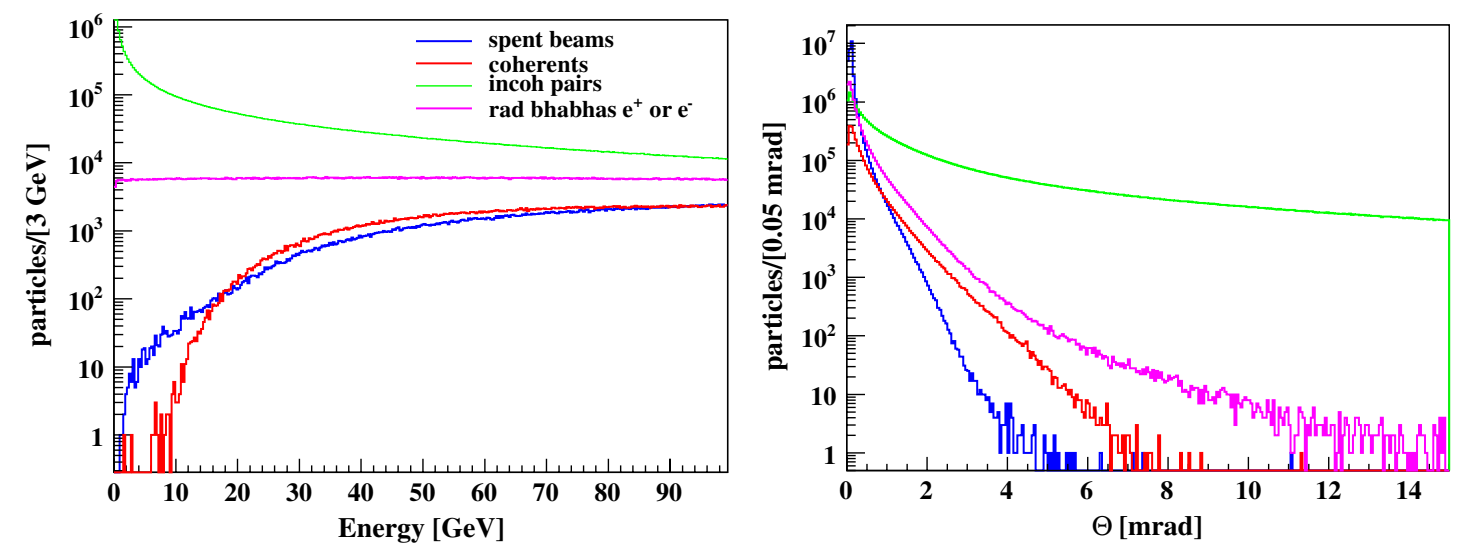

FIG. 1. Energy distribution up to $100 \mathrm{GeV}$ of the spent beam and the different beam-beam interaction products (left). Angular distribution at the interaction point of the spent beam and the different beam-beam interaction products (right). The distributions are not normalized with respect to their rates.

techniques studied. The beam-beam background processes and their correlations with luminosity are studied, considering several beam aberrations at the IP. Finally, a new potential signal from the $\gamma \gamma \rightarrow$ hadrons process is assessed for tuning purposes.

\section{BDS TUNING}

In order to mitigate effects due to static and dynamic imperfections, the CLIC BDS lattice (with $L^{*}=3.5 \mathrm{~m}$ ) is designed to reach a peak and total luminosity higher than their nominal values, by $\sim 20 \%$ and $\sim 30 \%$, respectively. The peak luminosity is defined as the luminosity in a region of $\pm 1 \%$ around the $3 \mathrm{TeV}$ peak. Half of the $20 \%$ peak luminosity is the budget for the static imperfections, the other half for the dynamic errors.

Table I shows the values of the peak and total luminosities obtained by tracking the beams in the ML and BDS (using the tracking code PLACET [11]) and computing them

TABLE I. Main parameters of CLIC at $E_{\mathrm{cm}}=3 \mathrm{TeV}$ accounting for imperfections. Nominal peak and total luminosity are in brackets. The background is per bunch crossing, beamstrahlung photons are per beam particle.

\begin{tabular}{lcc}
\hline \hline Total luminosity (nominal) $\mathcal{L}$ & {$\left[10^{34} \mathrm{~cm}^{-2} \mathrm{~s}^{-1}\right]$} & $7.7(5.9)$ \\
Peak luminosity (nominal) $\mathcal{L}$ & {$\left[10^{34} \mathrm{~cm}^{-2} \mathrm{~s}^{-1}\right]$} & $2.4(2.0)$ \\
Repetition frequency $f_{r}$ & {$[\mathrm{~Hz}]$} & 50 \\
Bunches per train $N_{b}$ & & 312 \\
Distance between bunches $\Delta_{y}$ & {$[\mathrm{~ns}]$} & 0.5 \\
Particles per bunch $N$ & {$\left[10^{10}\right]$} & 0.372 \\
Bunch length $\sigma_{z}$ & {$[\mu \mathrm{m}]$} & 44 \\
Emittances $\gamma \epsilon_{x} / \gamma \epsilon_{y}$ & {$[\mathrm{~nm}] /[\mathrm{nm}]$} & $660 / 20$ \\
Beam sizes $\sigma_{x}^{*} / \sigma_{y}^{*}$ & {$[\mathrm{~nm}] /[\mathrm{nm}]$} & $45 / 1$ \\
Beamstrahlung photons/particle $n_{\gamma}$ & & 2.1 \\
Incoherent pairs $N_{\text {pairs }}$ & {$\left[10^{3}\right]$} & 330 \\
Coherent pairs $N_{\text {coh }}$ & {$\left[10^{7}\right]$} & 33 \\
Hadronic events $N_{H}$ & $E_{\gamma \gamma_{\mathrm{cm}}}>5 \mathrm{GeV}$ & 2.8 \\
\hline \hline
\end{tabular}

with GUINEA-PIG [12], the corresponding nominal values are reported in parenthesis. The main beam parameters and the corresponding background rates are reported in Table I as well. In the following we present the first results of the tuning of the static imperfections in the CLIC BDS. Detailed studies for dynamic imperfections can be found in [13].

In the simulations we assume that all the magnets of the BDS or FFS (except for the bending magnets) are randomly displaced in the two transverse planes ( $x$ and $y$ ) with a Gaussian distribution of $\sigma=10 \mu \mathrm{m}$, which is defined to be the prealignment tolerance for this study. This value is a first estimate, which is very close to the value used in the main linac alignment [14]. In this study identical $e^{+} e^{-}$ machines are simulated. The three techniques studied to recover from the magnets displacements are: beam based alignment (BBA), of the entire BDS, combined with FFS sextupole knobs; luminosity optimization of the FFS; the latter combined with sextupole knobs.

The BBA technique consists of the 1-to-1 correction [15] followed by dispersion-free steering (DFS) [16] in the vertical plane and target DFS in the horizontal one. In the 1-to-1 correction, the beam is steered through the center of the beam position monitors (BPMs). DFS is a technique that measures the dispersion along the line, using off-energy test beams, and corrects it to zero or to the nominal value. An energy difference of $0.1 \%$ is used to measure dispersion. The assumed BPM resolution in these simulations is $10 \mathrm{~nm}$. This value is a first estimate of the required BPM resolution.

The possibility to use tuning knobs based on linear combinations of sextupole displacements has been already explored in CLIC [9]. New tuning knobs are built here using FFS sextupole displacements, in order to control mainly coupling, dispersion, and waist shift in the two transverse planes. The five sextupoles of the FFS are used to construct 10 linear knobs by their horizontal and vertical displacements. Assuming the BDS without errors, 

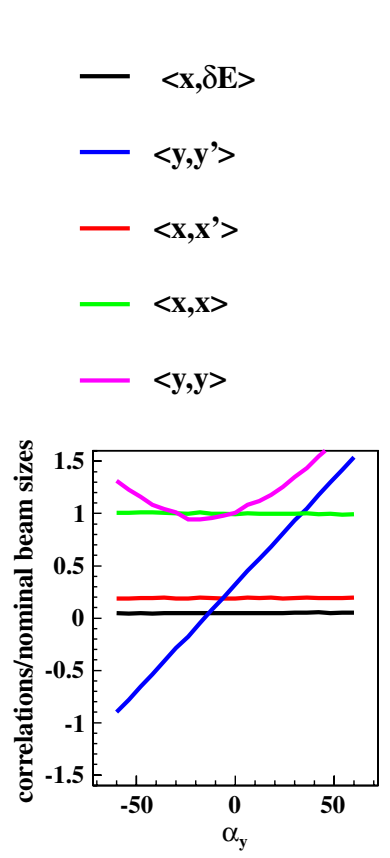
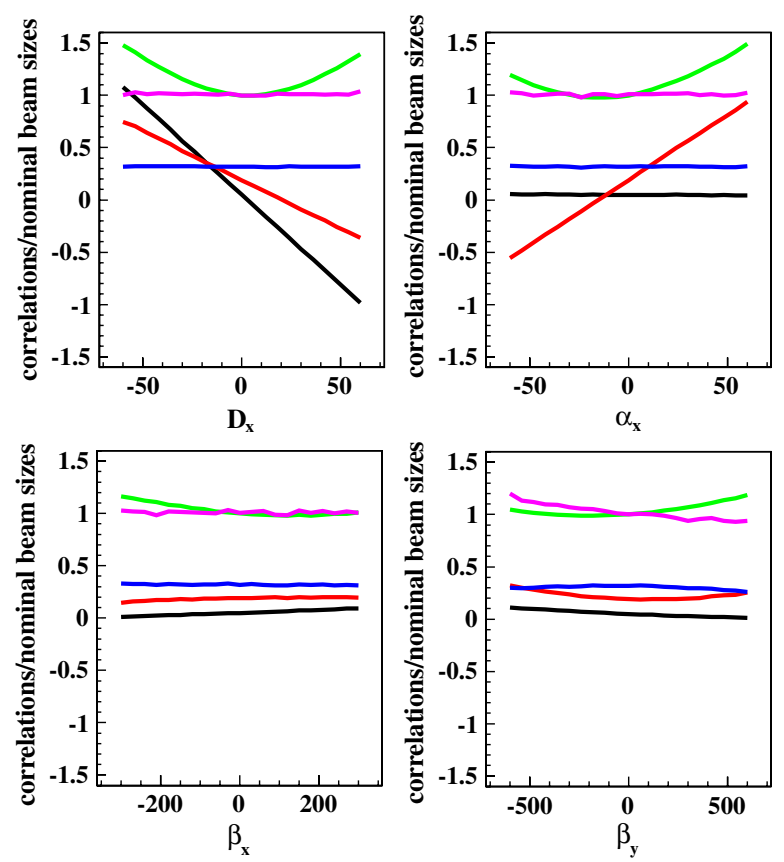

FIG. 2. Beam covariances at the IP as a function of the horizontal knobs scan. The range of the scan in units of $\mu \mathrm{m}$ is shown in Fig. 6 .

a single sextupole is moved by $0.1 \mu \mathrm{m}$ at a time. For each sextupole displacement the variations of the chosen set of aberrations are evaluated, and corresponding $5 \times 5$ response matrices $M_{x}$ and $M_{y}$ are built. The knobs correspond to the columns of the inverted matrices. The chosen set of knobs used to build $M_{x}$ are: the two $\beta$ at the IP, the two $\alpha$ and horizontal dispersion. The knobs in $M_{y}$ are $\langle x, y\rangle,\left\langle x^{\prime}, y\right\rangle,\left\langle x^{\prime}, y^{\prime}\right\rangle$, vertical dispersion, and vertical angular dispersion. These sets of knobs control the spectrum of the expected beams aberrations. Figures 2 and 3 show the effects of the knobs on the beam covariances at the IP, when they are scanned individually.

Despite the orthogonality condition with which they are built, these first knobs show a multiknobs behavior, whereby the knob constructed to control one aberration can act on another one. For example, the $\beta$ knobs that change the $\alpha\left(\left\langle x, x^{\prime}\right\rangle\right.$ and $\left.\left\langle y, y^{\prime}\right\rangle\right)$ at the IP as well. Nevertheless, all the knobs contribute to the overall
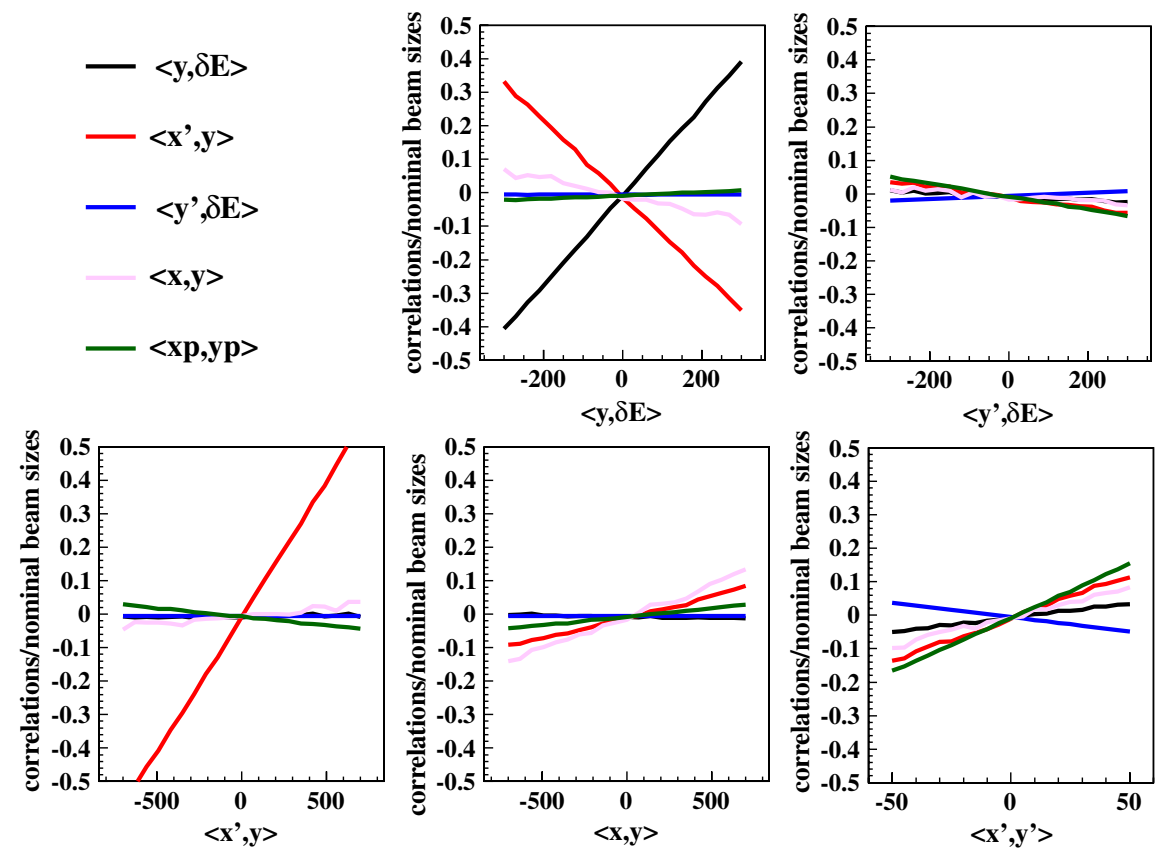

FIG. 3. Beam covariances at the IP as a function of the horizontal knobs scan. The range of the scan in units of $\mu \mathrm{m}$ is shown in Fig. 6. 


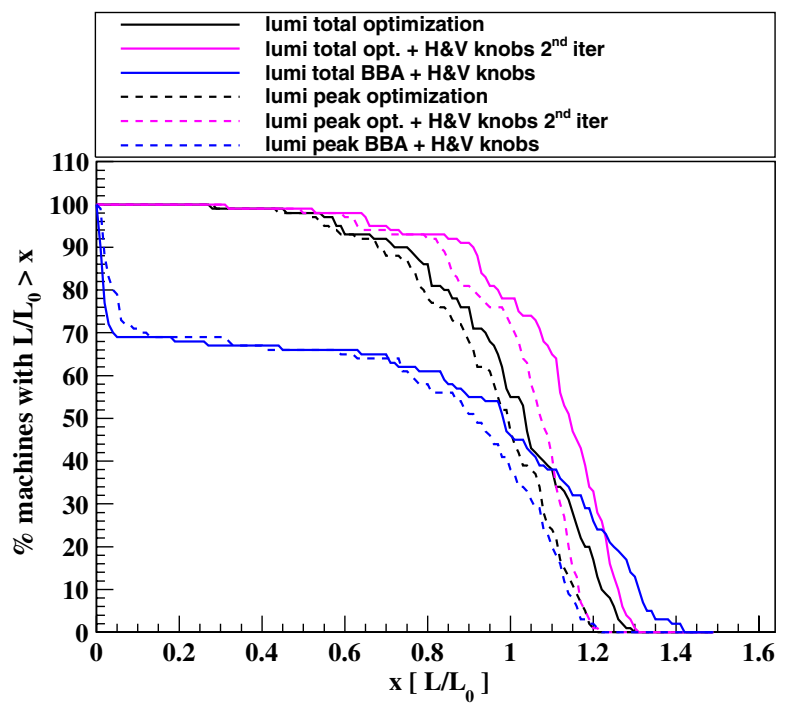

FIG. 4. Luminosity distribution of 100 machines after three different alignment procedures starting from an initial random prealignment of $10 \mu \mathrm{m}$. Full lines are the total luminosity values, dashed lines for the peak luminosity.

optimization procedure giving an increase of luminosity each time they are scanned. An optimization of such knobs to improve their orthogonality is planned for the future.

In the luminosity optimization procedure, the horizontal and vertical positions of the misaligned elements of the FFS (except for the bending magnets) are changed in order to maximize luminosity, using the Nedler-Mead algorithm (Simplex).

The results of the tuning of the BDS against magnet displacements are summarized in Fig. 4 for 100 machines and the most significant numerical values reported in Table II.

The BBA technique has proven successful in the CLIC collimation section alone, while it fails when the FFS is also considered [3]. Iterations of BBA combined with tuning knobs improve the correction. The final total and peak luminosity obtained after fifth iterations of BBA and tuning knobs for the 100 machines are shown in Fig. 4 (blue line). About $35 \%$ of the machines reach $10 \%$ more luminosity than the nominal value, which is

TABLE II. Number of machines reaching the target luminosity for the static imperfections in the three combinations of techniques here studied.

\begin{tabular}{lcc}
\hline \hline Technique & \# machines & \# machines \\
\hline & {$[110 \%$ of } & {$[110 \%$ of } \\
& total $\mathcal{L}]$ & peak $\mathcal{L}]$ \\
BBA + knobs & 38 & 20 \\
Luminosity optimization & 36 & 24 \\
Luminosity optimization + knobs & 65 & 40 \\
\hline \hline
\end{tabular}

the budget for static imperfections in the BDS. Of these, about $15 \%$ exceed the $30 \%$ more total luminosity we expect by design, while this is not the case for the peak luminosity. This effect is explained by the smaller horizontal beam size that is reached after the BBA and FFS knobs scan with respect to the nominal value. This causes, on one hand, the enhancement of total luminosity, and, on the other hand, the emission of more beamstrahlung photons with the consequent increase of average energy loss that smears the luminosity spectrum in the energy peak. With the luminosity optimization technique alone 36 machines reach the target of $10 \%$ more luminosity than nominal value. The best tuning performance is obtained by combining luminosity optimization and FFS knobs. In this case more than $60 \%$ of the machines reach the target value. It is worth noticing that about $90 \%$ of the machines reach $90 \%$ of CLIC nominal total luminosity.

Figure 5 shows the distribution of the number of iterations required by the techniques presented in Fig. 4. The iterations correspond to the number of luminosity measurements required. The number of luminosity measurements needed by the luminosity optimization procedure is 1 order of magnitude larger than the one required by the BBA combined with the Knobs technique. It is therefore crucial for CLIC to be able to measure luminosity as fast as possible (in the order of seconds) and to be able to tune the system in the most efficient way. The use of more sophisticated optimization algorithms and nonlinear knobs could improve the overall luminosity results and reduce the number of luminosity measurements required.

In the following, we concentrate on the definition of fast luminosity signals. For this purpose the beam-beam background processes and their correlation with the main sources of luminosity degradation are presented.

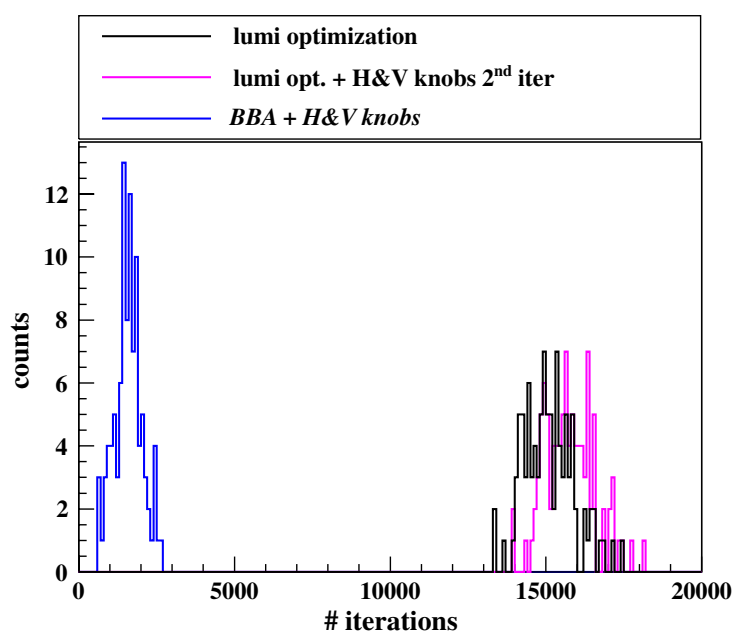

FIG. 5. Number of luminosity measurements required in the three different alignment procedures in order to reach the results shown in 4. 


\section{BEAM-BEAM BACKGROUND}

Because of the high energy and high luminosity foreseen at CLIC the background rates due to the beambeam interaction are expected to be high. The relevant processes are: the emission of beamstrahlung photons, the coherent pair production, the incoherent pair production, and the hadronic events. A detailed description of those processes can be found in [17].

Their expected rates in CLIC at $3 \mathrm{TeV}$ c.m. energy are reported in Table I. The actual values of luminosity and background depend on the single machine and can change during operation. A first study of the variation of the background rates with the offsets of the two beams and the emittance growth has been presented in [18]. All the background rates show the same behavior in case of horizontal offsets and horizontal emittance growth of the two beams at the IP, following luminosity variations. In the case of vertical offsets and vertical emittance growth, the coherent processes show a different behavior with respect to luminosity. These processes are due to the interaction of photons with the electromagnetic field produced by the bunches, which is strongly dominated by the larger horizontal beam size and increase with small vertical offsets, as shown in [18]. In the following we show in more detail the dependence of the backgrounds and luminosity on the specific beam aberrations at the IP. These are the same aberrations we used to define the sextupole knobs in the previous section.

\section{Luminosity signals and colliding beam parameters}

We present here a study of the variation of different signals from beam-beam interaction, according to 10 different beam aberrations at the IP. The size of the aberrations has been chosen to produce a luminosity loss of about $30 \%$. The six Signals $(S)$ we have defined are: (i) coherent pairs from the two beams (coherent); (ii) average beamstrahlung photons from the two beams $\left[\left(n_{\gamma 1}+n_{\gamma 2}\right) / 2\right]$; (iii) difference of beamstrahlung photons from the two beams normalized to their sum $\left(1.0-\left|\Delta n_{\gamma} / \Sigma n_{\gamma}\right|\right)$; (iv) ratio of beamstrahlung photons from the two beams $\left(n_{\gamma 2} / n_{\gamma 1}\right)$; (v) total number of $\gamma \gamma \rightarrow$ hadrons events (hadrons); (vi) total incoherent pairs from the two beams (incoherent).

The two beams are transported to the IP as described in the previous section. In this case the sextupoles of one beam line are displaced according to the linear knobs introduced in Sec. II, generating the beam phase space distortion at the IP. The second beam line instead is kept perfectly aligned.

Figure 6 shows the relative change of the six signals and of the peak and total luminosity by scanning the five horizontal knobs. The colored lines correspond to parabolic fits through the data points. The optimum luminosity values coming from the parabolic fits of the six signals reproduce the maximum value of luminosity with a relative accuracy of the order of $10^{-3}$. The numerical uncertainties are about $2 \%$ in the case of incoherent pairs and hadronic events and about $6 \%$ in
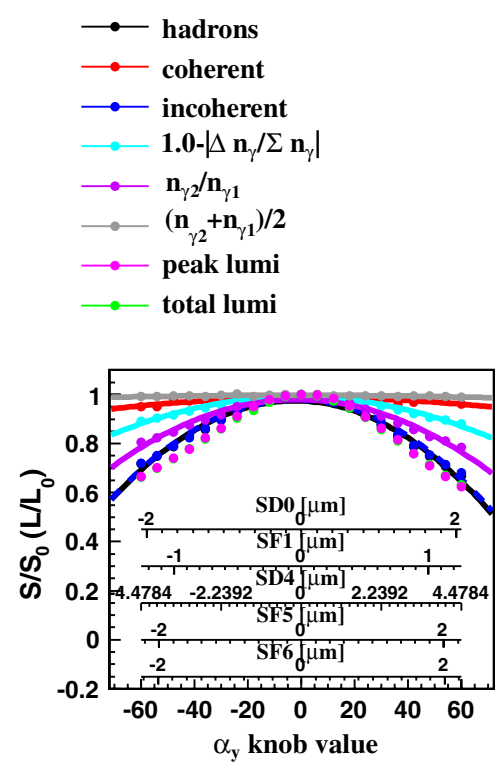
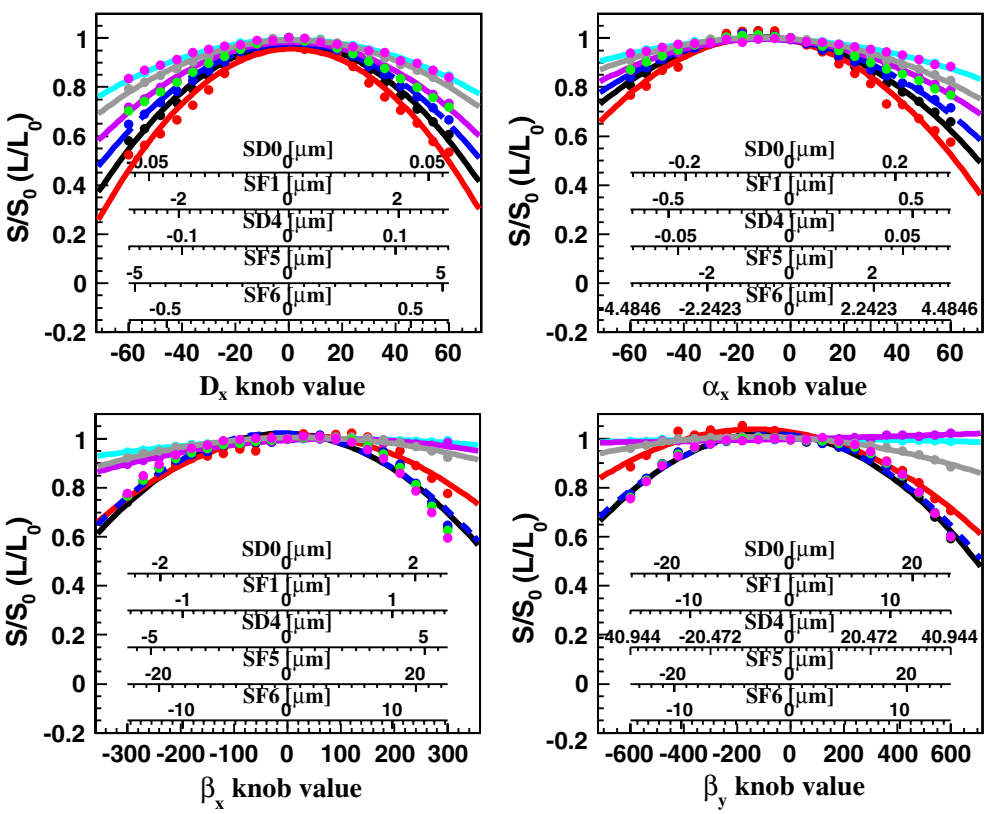

FIG. 6. Scan of the horizontal tuning knobs. The points show the change of luminosity $\left(L / L_{0}\right)$ and beam-beam background $\left(S / S_{0}\right)$ rates (normalized to their nominal values), according to horizontal dispersion, $\alpha$ and $\beta$ functions in the two planes. The lines represent the fit through the background rates points. The lines corresponding to the fit through the points of the total and peak luminosity are not shown. 

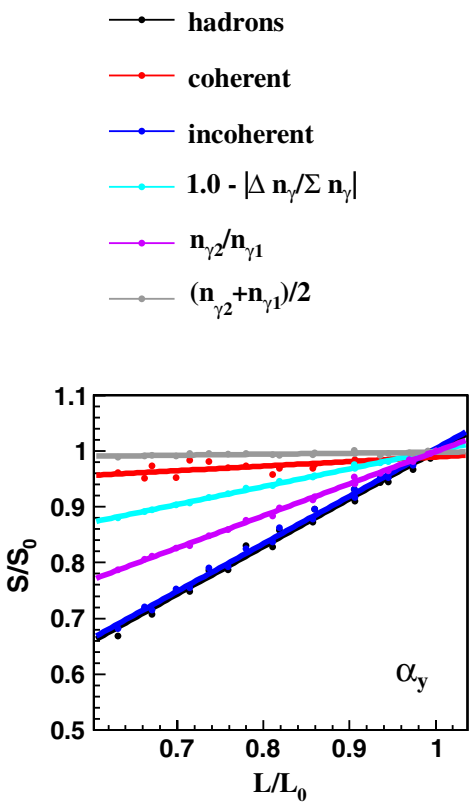
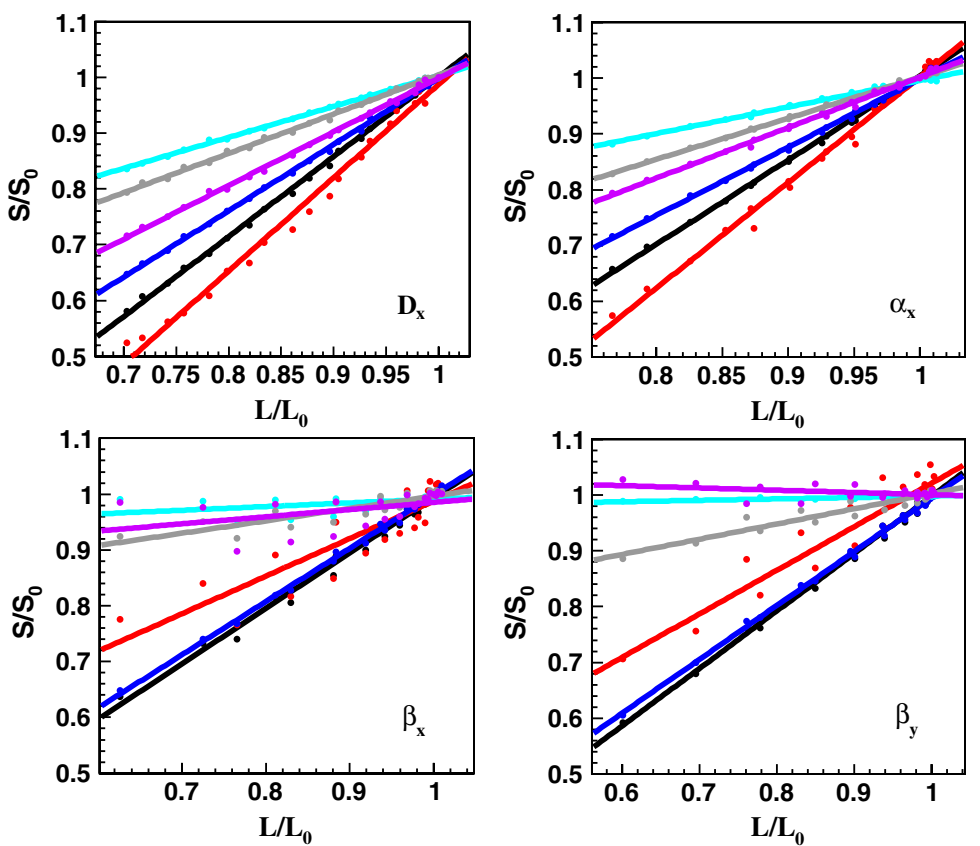

FIG. 7. Beam-beam background signal correlation with total luminosity for the scan of the five horizontal knobs shown in Fig. 6.

the case of beamstrahlung and coherent processes. Figure 7 shows a fairly linear correlation of the six signals to the total luminosity in this range of scan of the knobs. The angular coefficients [that we name $\left.\Delta\left(S / S_{0}\right) / \Delta\left(L / L_{0}\right)\right]$ obtained from the linear fits of the data points are shown in Fig. 8. A value of $\Delta\left(S / S_{0}\right) / \Delta\left(L / L_{0}\right)$ equal or close to 1 means that the variation of the luminosity and of the corresponding background signal are equal. The incoherent pairs signal presents an angular coefficient close to 1 (within $20 \%$ uncertainty) for all five horizontal aberrations considered. The relative hadronic events rate shows the same behavior as the incoherent pairs except for

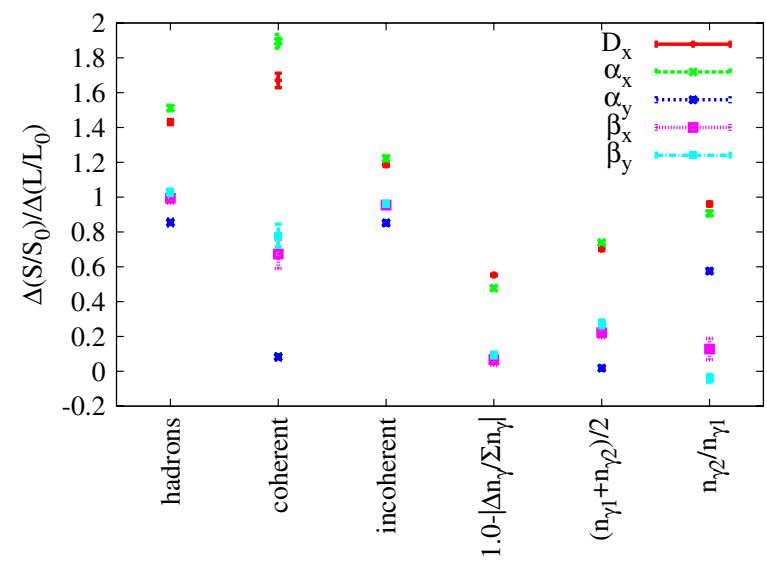

FIG. 8. Relative change of the six signals with total luminosity coming from the angular coefficient of the fit shown in Fig. 7. horizontal dispersion and horizontal waist shift. In these two cases in fact the horizontal beam size increase leads to the reduction of beamstrahlung photons, hence to the decrement of $\gamma$ available for the $\gamma \gamma \rightarrow$ hadrons reaction. The relative change of these two types of processes follow the luminosity variation within $20 \%$ uncertainty independently of the beam aberrations.

Figures 9-11 show the same analysis, when we scan the five vertical knobs. The behavior of background signals vs luminosity is similar to the horizontal case.

In summary, the incoherent processes, such as incoherent pairs and hadronic events, have the same variation of luminosity within $20 \%$ (i.e., the correlation between the change of these event rates and the change of luminosity is close to 1 ), regardless of the aberrations considered. The coherent processes, such as coherent pairs and beamstrahlung, have a different variation with respect to luminosity (i.e. their correlation is far from 1) and present a different variation according to the aberrations too. Therefore, by measuring the variation of the rates of the incoherent processes it is possible to evaluate the variation in the luminosity with $20 \%$ uncertainty. The variation of the rates of coherent processes could be used in combination with the one of incoherent processes to identify the main aberrations of the beams, in dedicated feedback.

In practice, it is critical to define a signal that can be easily identified against the other processes. Experimental techniques to detect beamstrahlung photons in the CLIC post collision line can be found in [19]. The incoherent pairs are produced with relatively small angles with respect to the 

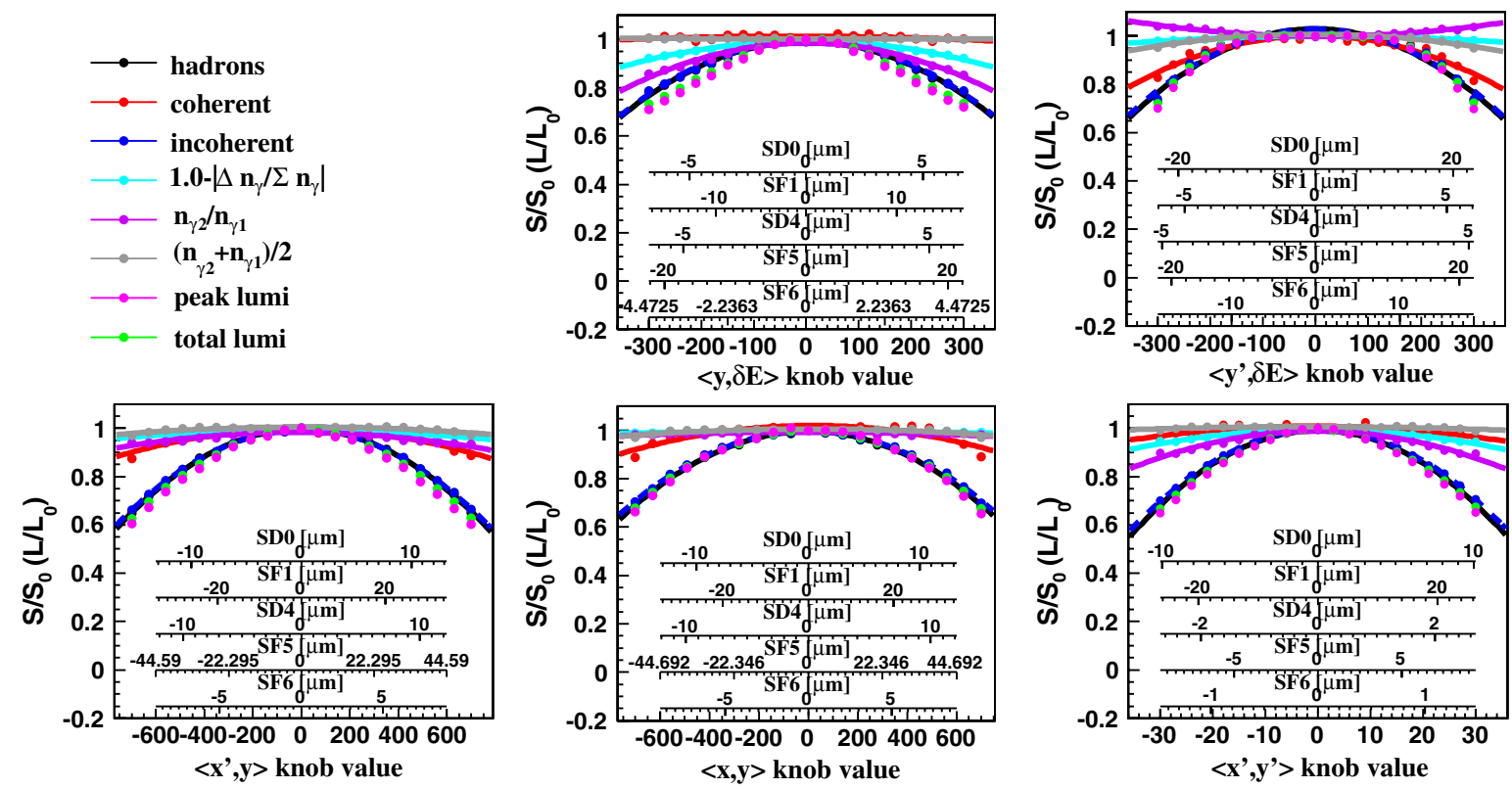

FIG. 9. Scan of the vertical tuning knobs. The points show the change of luminosity $\left(L / L_{0}\right)$ and beam-beam background $\left(S / S_{0}\right)$ rates (normalized to the nominal value), according to vertical dispersion, angular dispersion, and couplings. The lines represent the fit through the background rate points. The lines corresponding to the fit through the points of the total and peak luminosity are not shown.
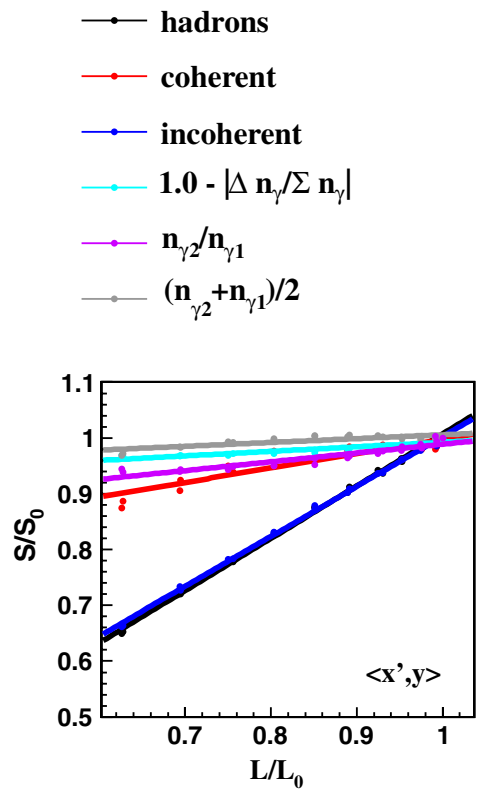
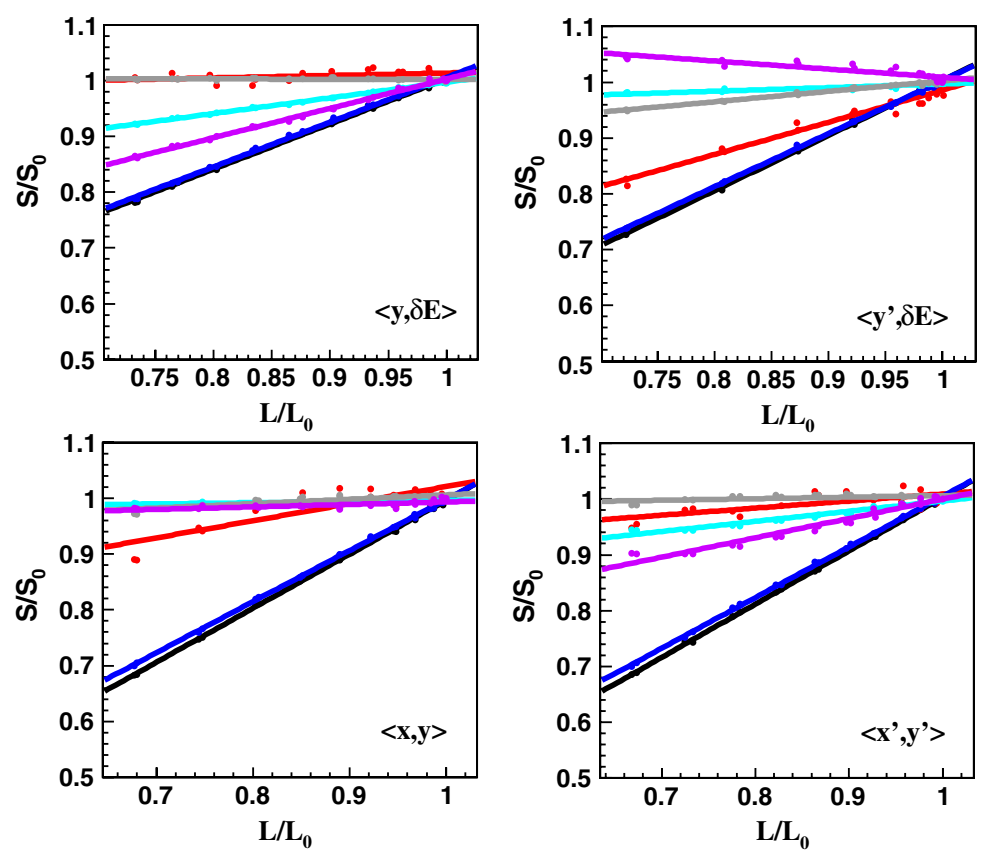

FIG. 10. Beam-beam background signal correlation with total luminosity for the scan of the five vertical knobs shown in Fig. 9.

beam axis, but are deflected by the beam fields. Therefore, the pair particles can have large angles. The integration of pair energy above a certain angle with respect to the beam axis has been studied as a potential signal for luminosity optimization in [8]. In CLIC their identification could be more complicated due to the presence of the coherent pairs in the forward region, leptons coming from hadronic events, and Bhabhas. In the following we discuss further the hadronic events signal by looking in particular at its multiplicity in the final state of the process. 


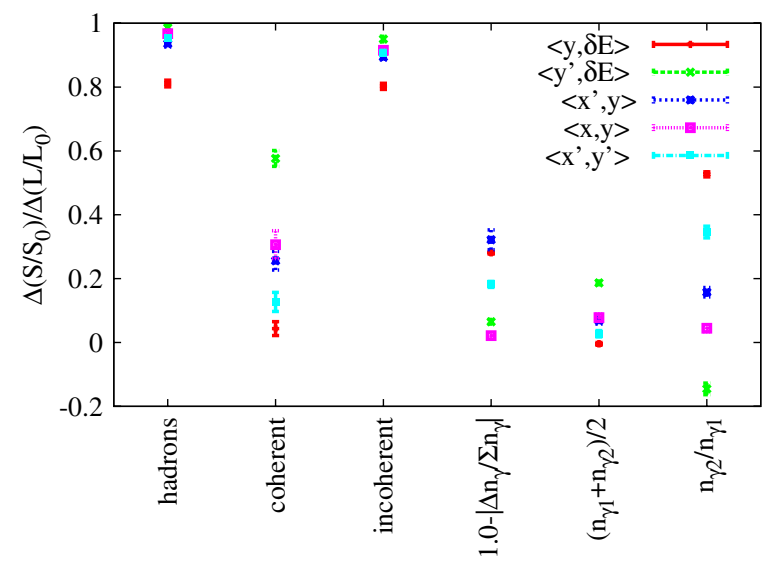

FIG. 11. Relative change of the six signals with total luminosity coming from the angular coefficient of the fit shown in Fig. 10.

\section{HADRONIC EVENTS}

Hadrons at linear colliders are produced by the process $e^{+} e^{-} \rightarrow \gamma \gamma \rightarrow$ hadrons. The total $\gamma \gamma \rightarrow$ hadrons cross section is known experimentally up to $200 \mathrm{GeV}$ in the center of mass energy. The simplest model of the energy dependence of the $\gamma \gamma \rightarrow$ hadrons cross section $(\sigma)$ is the vector meson dominance one. The model assumes that the photon resonates to a hadronic state (a $\rho$ ) with a certain probability [20], with the energy dependence expressed as

$$
\sigma=211 \mathrm{nb} \cdot\left(\frac{s}{\mathrm{GeV}}\right)^{\epsilon}+215 \mathrm{nb} \cdot\left(\frac{s}{\mathrm{GeV}}\right)^{\mu}
$$

where $\epsilon=0.0808$ and $\mu=0.4525$ [21]. GUINEA-PIG implements the above parametrization of the total $\gamma \gamma \rightarrow$ hadrons cross section. An electron or positron is replaced by the appropriate number of photons from the equivalent spectrum. The energies of the two colliding photons can be stored in a file which can be loaded as input to PYTHIA [22], or an equivalent code, to generate the hadrons.
Detectable signals for this process can be the hadron multiplicity and/or their deposited energy in the detector region. The total and single particles average multiplicity in the final state of the $\gamma \gamma \rightarrow$ hadrons collision as shown in Fig. 12 (left). The average total multiplicity is dominated by the photons and charged pions, while the leptons and the other hadrons multiplicity is close to zero in all the events analyzed. Figure 12 (right) shows the total energy distribution. In order to define a region where the hadron multiplicity can be easily identified and detected against the other background sources, two different $p_{T}$ cuts are applied to the charged particles in order to ensure that they can travel in the forward detector region or in the detector main tracking region, considering a $B$ field of 5 Tesla. Following [23], we consider tracks with $p_{T}>0.050 \mathrm{GeV}$ and $27 \mathrm{mrad}<\theta<117 \mathrm{mrad}$ for the forward region and tracks with $p_{T}>0.160 \mathrm{GeV}$ and $117 \mathrm{mrad}<\theta<$ $1.57 \mathrm{rad}$ for the main tracking region.

The $27 \mathrm{mrad}$ condition for the forward region is due to the envelope of the incoherent pairs while traveling in the detector solenoid magnetic field. We track the particles with a helix up to different longitudinal positions, taking into account the CLIC crossing angle. The incoherent pair particle positions in the $X Y$ plane, at $z=2.65 \mathrm{~m}$ from the IP, are shown in Fig. 13 (left). A circle containing the majority of the particles is defined "by eye" at each longitudinal position. The points so determined and the definition of the two angular cuts are shown in Fig. 13 (right).

The resulting multiplicity distributions of all the charged particles and that of the hadrons, according to the selected angles and momenta, are shown in Fig. 14. The multiplicities integrated over 1, 10, and 20 trains are shown. Almost all the multiplicity from $\gamma \gamma$ collision consists of charged hadrons. The mean value of the distribution over 20 trains is determined with about $1 \%$ fluctuation. Taking into account the $20 \%$ uncertainty in the correlation of this signal with luminosity, as shown in Figs. 8 and 11, this gives an estimation of luminosity variations within $1.2 \%$ precision. Moreover, given the CLIC repetition rate

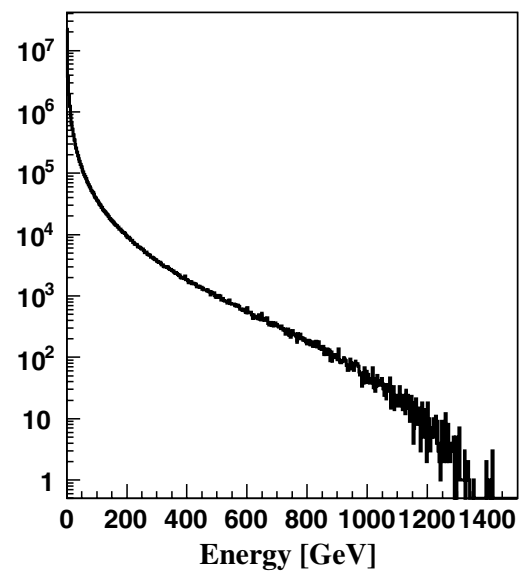

FIG. 12. Average particles multiplicity and energy for a single $\gamma \gamma$ collision. 

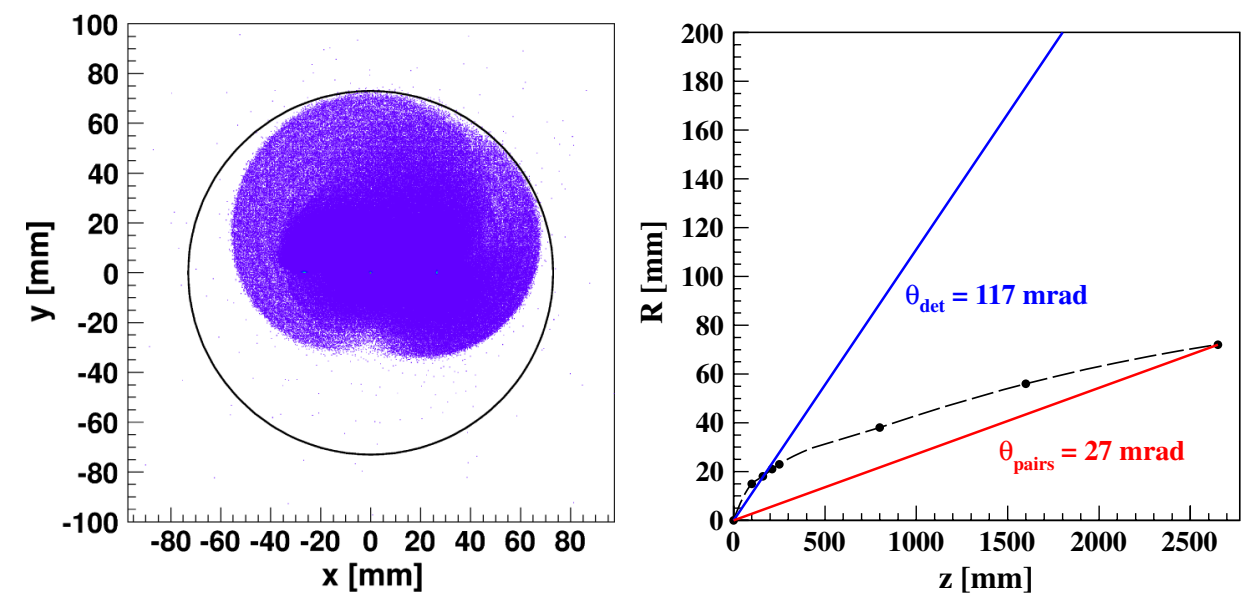

FIG. 13. Incoherent pair particle positions, at $z=2.65 \mathrm{~m}$ from the IP, for a constant $B$ field of $5 \mathrm{~T}$ (left). Definition of the two regions for the $\gamma \gamma \rightarrow$ hadrons multiplicity detection (right). The blue line represents the main tracking region acceptance. The black points and the dashed line represent the incoherent pairs envelop. The red line is the angular cut we define to separate the hadronic events from the incoherent ones.
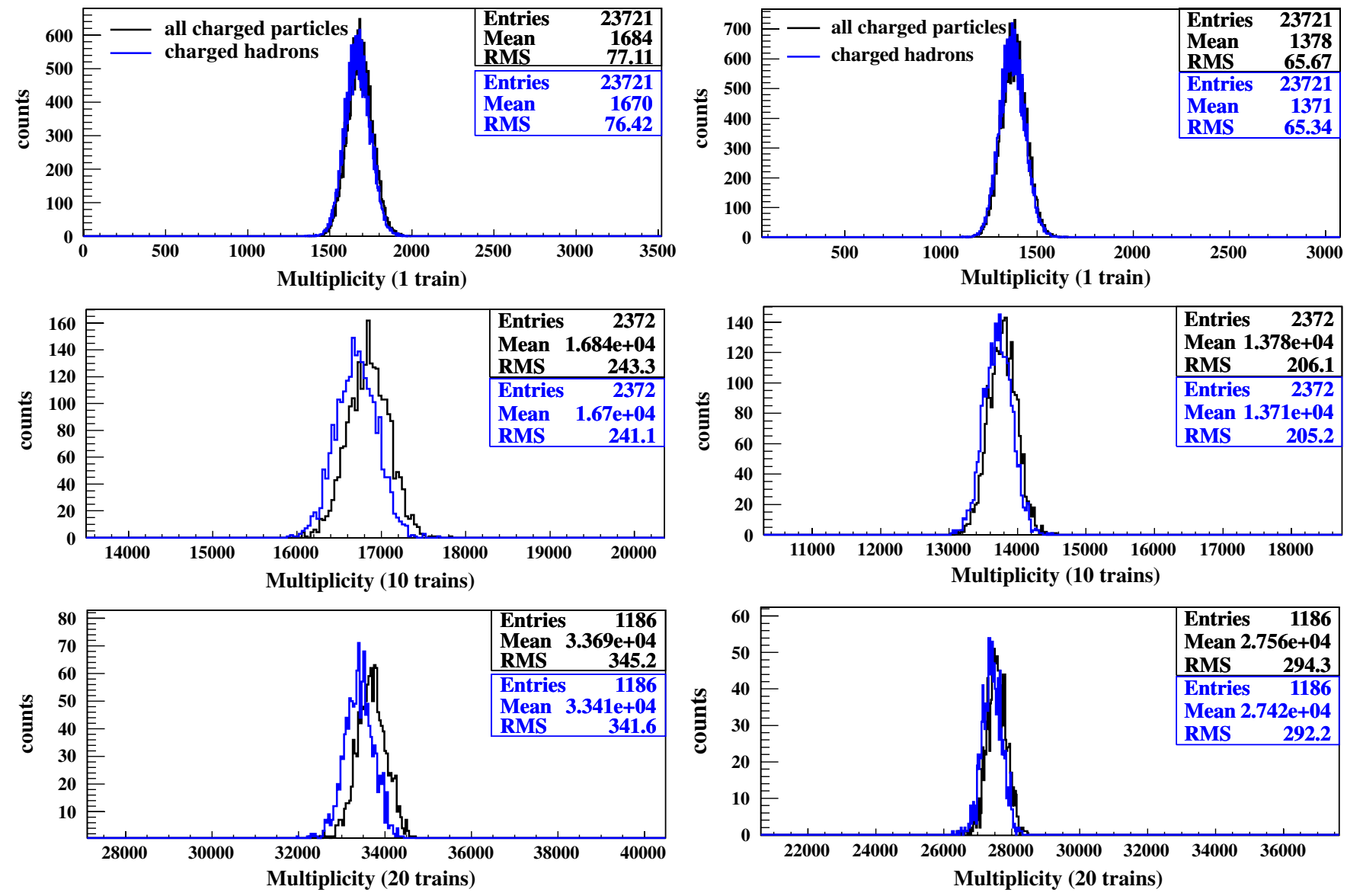

FIG. 14. Integrated charged particles and charged hadrons multiplicity over 1,10 , and $20 \mathrm{CLIC}$ trains with $p_{T}>0.050 \mathrm{GeV} / c$ and $0.027<\theta<0.117 \mathrm{rad}$ (left), and with $p_{T}>0.160 \mathrm{GeV} / c$ and $0.117<\theta<1.57 \mathrm{rad}$ (right). 

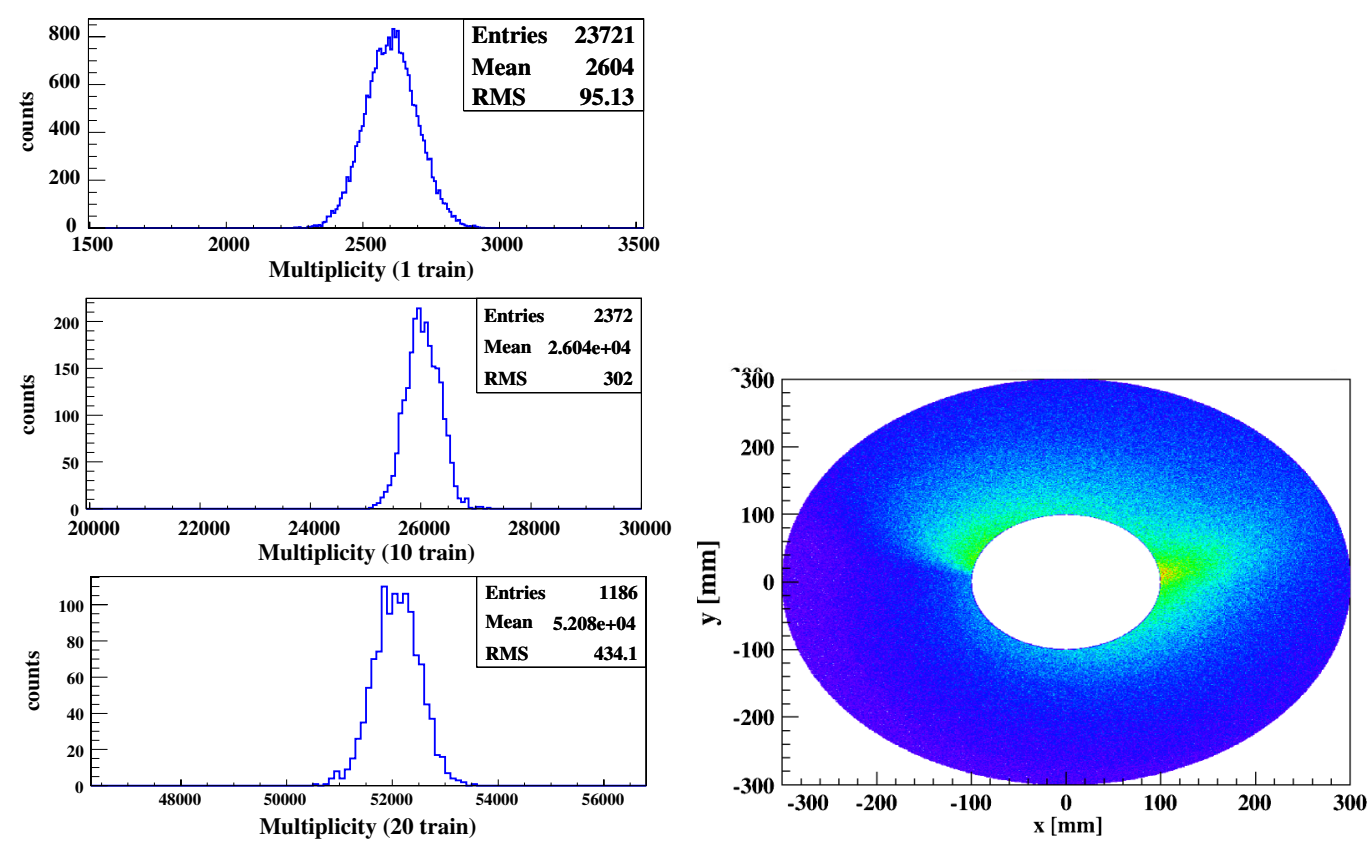

FIG. 15. Integrated particle multiplicity over 1, 10, and 20 CLIC trains in a disk of inner radius of $10 \mathrm{~cm}$ and outer radius of $30 \mathrm{~cm}$ at $2.6 \mathrm{~m}$ from the IP. The scatter plot of the $X, Y$ positions of the particles in the defined disk is shown on the right.

$(50 \mathrm{~Hz}) 20$ trains correspond to 0.4 seconds. Requiring a total time $\leq 0.1 \mathrm{~s}$ for the readout electronics and signal elaboration, one luminosity measurement should take about $0.5 \mathrm{~s}$.

Considering the number of particles intercepting a disk of $10 \mathrm{~cm}$ inner radius and $30 \mathrm{~cm}$ outer radius at $2.6 \mathrm{~m}$ from the IP, the rms fluctuation on the mean is of the order of $1 \%$ integrating over 10 trains only [Fig. 15 (left)]. This corresponds to about $0.3 \mathrm{~s}$ for one luminosity measurement. The two-dimensional distribution of the $x$ and $y$ positions at the disk (after the tracking in a uniform magnetic field of 5 Tesla) is shown in Fig. 15 (right). Taking into account the number of iterations that requires a luminosity measurement in the procedures studied here, the total time needed to tune the BDS, starting from a random Gaussian displacement of the FFS magnet of $10 \mu \mathrm{m}$, would be about 10 min when the BBA technique in combination with the FFS sextupoles knobs are applied, even with low success rate. The total time required to tune the BDS with the luminosity optimization technique is instead of the order of 2 hours. The full CLIC detector model is not considered in these simulations. The actual amount of material and the interaction of these particles with matter should be considered in order to define the best region of detection of the multiplicity minimizing the number of bunches to be integrated in the definition of the trigger signal.

\section{CONCLUSIONS}

In order to mitigate the static imperfections in the CLIC BDS different techniques have been applied. The first results show that their combination gives $90 \%$ of CLIC nominal total luminosity in $90 \%$ of the cases. Among them $65 \%$ reach the target luminosity of $110 \%$. In particular, for the first time sextupole knobs are successfully and systematically used within CLIC FFS tuning. Tuning knobs and the luminosity optimization technique require a fast luminosity measurement. For this purpose, the possibility to use $\gamma \gamma \rightarrow$ hadrons background has been investigated. The first study of the charged particle multiplicity from this process in the vertex-tracking and/or in the forward region of the detector shows that it could provide a signal for a fast luminosity measurement in less than $1 \mathrm{~s}$ with $\sim 1 \%$ precision. Given the number of luminosity measurements needed by the different alignment techniques here considered, the full tuning of the CLIC BDS against magnet displacements can be achieved in the range between about 10 min and 2 hours.

[1] J. Pfingstner and J. Snuverink (to be published); CLIC Conceptual Design Report (CDR) [http://project-cliccdr.web.cern.ch/project-CLIC-CDR].

[2] G. White et al., in Proceedings of the International Particle Accelerator Conference, Kyoto, Japan (ICR, Kyoto, 2010), WEOBMH01

[3] A. Latina et al., Report No. CERN-AB-2008-011; Report No. CLIC-Note-753; Report No. EUROTeV-Report-2008040.

[4] R. Tomás, Phys. Rev. ST Accel. Beams 9, 081001 (2006).

[5] R. Tomás et al., Report No. CERN-ATS-2009-092; Report No. EuCARD-CON-2009-058; Report No. CLIC-Note792. 
[6] D. A. Karlen, Ph.D. thesis, Stanford University [Report No. SLAC-325, 1988].

[7] D. Schulte, at the CLIC 08 workshop at CERN, 2008.

[8] D. Schulte, Report No. CLIC CERN-AB-2004-068; Report No. CLIC-Note-600.

[9] P. Eliasson et al., Report No. CERN-AB-2006-044; CLIC Report No. 660; EUROTeV Report No. 2006-039.

[10] T. Tauki and K. Yokoya, Phys. Rev. E 51, 6119 (1995).

[11] D. Schulte et al., Report No. CERN/PS 2001/028.

[12] D. Schulte, CERN-PS-99-014-LP; CLIC-Note-387.

[13] J. Snuverink et al., in Proceedings of the International Particle Accelerator Conference, San Sebastian, Spain, 2011, TUPC023.

[14] D. Schulte et al., in Proceedings of the Particle Accelerator Conference, Vancouver, Canada, 2009, TH6PFP045 .

[15] Chris Adolphsen et al., in Proceedings of the 1989 Particle Accelerator Conference, Chicago, IL (IEEE, New York, 1989), p. 977.
[16] T. Raubenheimer and R. Ruth, Nucl. Instrum. Methods Phys. Res., Sect. A 302, 191 (1991).

[17] D. Schulte, Ph.D. thesis, University of Hamburg [Report No. TESLA 97-98, 1997].

[18] B. Dalena and D. Schulte, Reports No. CERN-ATS-2010080, CLIC-Note-835.

[19] R. B. Appleby, A. Apyan, L.C. Deacon, and E. Gschwendtner, in Proceedings of the International Particle Accelerator Conference, San Sebastian, Spain, 2011, TUPC027.

[20] P. Chien, T.L. Barklow, and M. E. Peskin, Phys. Rev. D 49, 3209 (1994).

[21] G. A. Schuler and T. Sjöstrand, Report No. CERN-TH/96119, 1996.

[22] T. Sjöstrand, S. Mrenna, and P. Skands, J. High Energy Phys. 05 (2006) 026.

[23] D. Dannheim and A. Sailer, CERN LCD-Note2011-021. 